\title{
Pseudoaneurysm of External Iliac Artery Following Septic Loosening of Total Hip Arthroplasty
}

\author{
P Rengsen, MBBS (Malaya), AA Abbas, MS (Ortho), SK Choon, FRCS (Eng), \\ CC Tai, FRCS (Tr \& Ortho) \\ Adult Reconstructive Surgery Unit, Department of Orthopaedic Surgery, University Malaya Medical Centre, \\ Kuala Lumpur, Malaysia
}

\begin{abstract}
The incidence of pseudoaneurysm after total hip arthroplasty is extremely rare. The most common mechanism of vascular injury is due to direct trauma during the operative procedure and most reported cases are acute in presentation. We report an unusual case of pseudoaneurysm of the external iliac artery, presenting 6 years after initial surgery, and occurring following septic loosening of total hip arthroplasty. This case highlights the importance of prompt recognition of infection of total hip arthroplasty, and also indicates the need for prompt and appropriate treatment for these infections.
\end{abstract}

Key Words:

Pseudoaneurysm, Total hip arthroplasty, Infection, External iliac artery

\section{INTRODUCTION}

Vascular injury secondary to hip surgery is uncommon in that the reported incidence of major vascular injury after surgical procedures on the hip is only $0.25 \%{ }^{1}$. The development of a pseudoaneurysm after total hip arthroplasty (THA) is an extremely rare complication. Most reported cases are acute in onset and are usually due to direct trauma during the operative procedure ${ }^{2}$. We report an unusual case of pseudoaneurysm of the external iliac artery in a patient with an infected THA, occurring 6 years after initial hip surgery.

\section{CASE REPORT}

A 48-year-old man was referred for further management of his left hip problem in 2006. He was diagnosed avascular necrosis of unknown origin in both hips when he was 38 years old. The patient subsequently underwent THA in the right and then the left hip (ten years and six years ago respectively). His surgical hip wounds healed well and there were no documented perioperative complications, and he was symptom free from both hips after surgery. However, over the three months prior to his recent admission, he complained of severe left groin pain, especially upon weight bearing, and had purulent discharge from his left surgical hip scar. Over the same period of time, the patient also noticed his lower abdomen had gradually swelled. His right hip remained symptom free.

Physical examination revealed a sinus with pus draining from the proximal portion of the previous left surgical hip scar. His left leg was shortened and externally rotated. Range of movement of the left hip was limited in all directions and painful on movement. There was a large pulsatile mass with an audible bruit in the lower abdomen which extended into his left groin. Distal pulses of the left leg were present.

The patient's haemoglobin at admission was $8.9 \mathrm{~g} / \mathrm{dl}$. Routine pelvic radiographs revealed osteolytic destruction of the acetabulum with cranial migration of the acetabular component of the THA, and protrusion of the implanted femoral head into the left hemipelvis (Fig. 1). A computed tomography (CT) scan showed a large multiloculated pseudoaneurysm around the implanted femoral head and acetabular component that measured $13.1 \times 9.6 \times 10.2 \mathrm{~cm}$. A subsequent arteriogram of the iliac vessels revealed a defect in the mid-portion of the external iliac artery, medial to the femoral head and acetabular component (Fig. 2). An endovascular graft vessel stent was then implanted transfemorally.

Removal of the hip implant was subsequently performed via lateral approach of the left hip. The hip implants were easily loosened and removed. There was extensive deep infection in the hip, and the posterior and medial acetabular walls were breached. Tissue specimens revealed infection with Staphylococcus aureus. An antibiotic impregnated cement spacer was inserted following thorough tissue debridement and washout. Re-implantation of new hip prosthesis was not performed at that time. The hip wound was partially closed and vacuum suction drainage was used at the surgical site. 


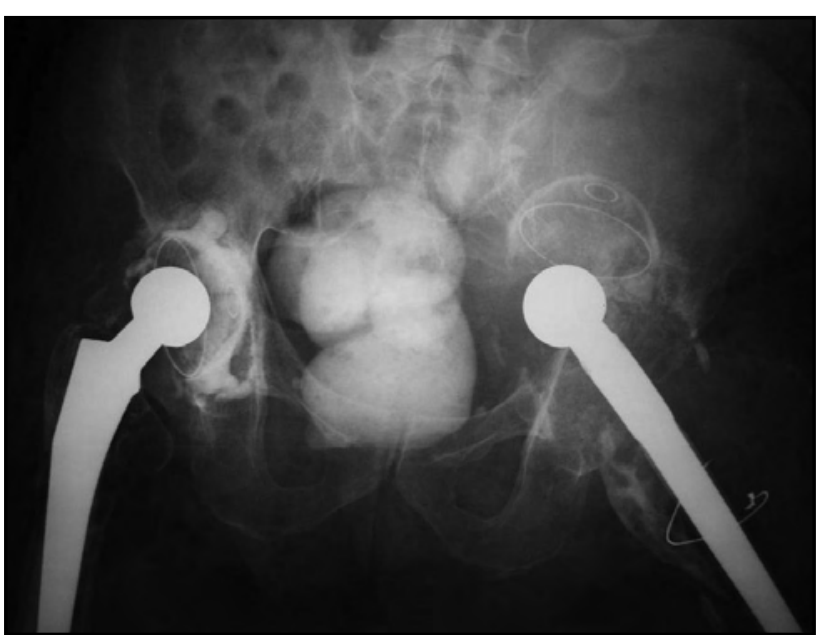

Fig. 1: Anteroposterior radiograph of the pelvis revealed cranial migration of the left acetabular component and protrusion of the left femoral head into the pelvis.

Appropriate antibiotic therapy was administered for six weeks postoperatively, and the patient recovered slowly during those six weeks. The wound healed without any sequelae within 3 weeks. He was pain-free after surgery and is currently awaiting revision total hip replacement.

\section{DISCUSSION}

Many reports of complications after THA have been published, but relatively little attention has been devoted to serious vascular injuries. In the English literature, the occurrence of pseudoaneurysm post THA is an extremely rare event. Most reported cases were acute in presentation and the average time interval between surgery and manifestation of the pseudoaneurysm was nine months (range, 20 hours to 23 months) ${ }^{3}$. The mechanism of vascular injury in most cases are due to direct trauma during the operative procedure such as perforation of vessels by retractors, osteotomes, powered reamers, screws, cement or even manoeuvres to dislocate hip ${ }^{1,2}$.

Unlike most reported cases, our patient first developed symptoms of pseudoaneurysm six years after initial surgery. The sequences of event most likely arose from septic loosening of the THA components thereby causing pseudoaneurysm due to intrapelvic migration of the acetabular and femoral head components. The other possibility is that the pseudoaneurysm was secondary to pyogenic staphylococcal sepsis. In either case, we believe that sepsis played an important role in the development of the pseudoaneurysm.

This complication might have been avoided by early recognition of septic loosening of the THA followed by

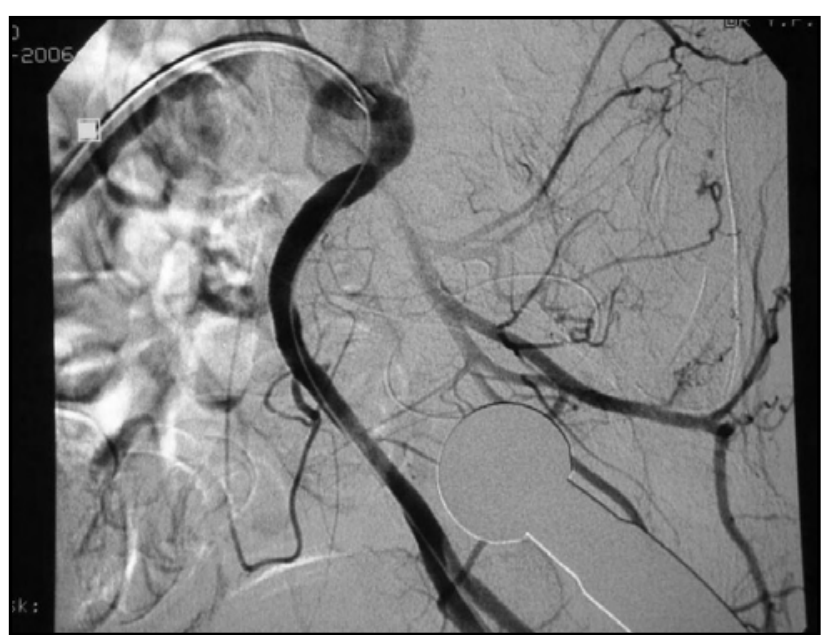

Fig. 2: A digitally enhanced and subtracted image of an angiogram of the external iliac artery showing a large pseudoaneurysm of the external iliac artery in close proximity to the acetabular component and implanted femoral head.

appropriate revision surgery to prevent migration of acetabular component. The case serves as a reminder that in THA revision, it is important to consider the possibility of the existence of an aneurysm or pseudoaneurysm, especially in cases where there is a migrated acetabular component.

Bach et $\mathrm{l}^{3}$ reported an injury to the external iliac artery from an acetabular cup which resulted in formation of a pseudoaneurysm. They believed that the threaded acetabular cup with sharp cutting flutes might have caused direct lesion of the arterial wall. Although the acetabular component used in this patient did not have sharp cutting flutes, we believe that the arterial lesions might have been similarly caused by repetitive direct trauma from either the acetabular cup or the implanted femoral head.

\section{CONCLUSION}

Vascular complications associated with THA are remarkably rare, making diagnosis and treatment of such sequelae extremely challenging for surgeons who are not familiar with their management (as evidenced by the high rate of limb loss, $70 \%$ ). This case demonstrates that a pseudoaneurysm does not always manifest as an acute presentation secondary to direct injury during a surgical procedure. It can present late, and be caused by repetitive trauma from arthroplasty components secondary to septic loosening. A pseudoaneurysm can threaten both the survival of the affected limb and the life of the patient. It is therefore important to recognise the early signs and symptoms of infected arthroplasty in order to prevent such complications. If a pseudoaneurysm is suspected, an angiogram should be performed followed by appropriate treatment as soon as possible. 


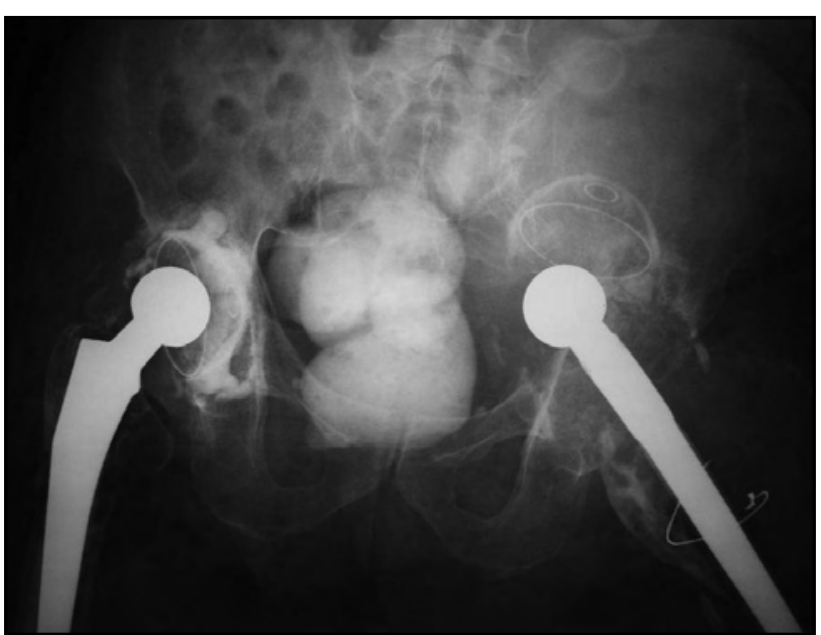

Fig. 1: Anteroposterior radiograph of the pelvis revealed cranial migration of the left acetabular component and protrusion of the left femoral head into the pelvis.

Appropriate antibiotic therapy was administered for six weeks postoperatively, and the patient recovered slowly during those six weeks. The wound healed without any sequelae within 3 weeks. He was pain-free after surgery and is currently awaiting revision total hip replacement.

\section{DISCUSSION}

Many reports of complications after THA have been published, but relatively little attention has been devoted to serious vascular injuries. In the English literature, the occurrence of pseudoaneurysm post THA is an extremely rare event. Most reported cases were acute in presentation and the average time interval between surgery and manifestation of the pseudoaneurysm was nine months (range, 20 hours to 23 months) ${ }^{3}$. The mechanism of vascular injury in most cases are due to direct trauma during the operative procedure such as perforation of vessels by retractors, osteotomes, powered reamers, screws, cement or even manoeuvres to dislocate hip ${ }^{1,2}$.

Unlike most reported cases, our patient first developed symptoms of pseudoaneurysm six years after initial surgery. The sequences of event most likely arose from septic loosening of the THA components thereby causing pseudoaneurysm due to intrapelvic migration of the acetabular and femoral head components. The other possibility is that the pseudoaneurysm was secondary to pyogenic staphylococcal sepsis. In either case, we believe that sepsis played an important role in the development of the pseudoaneurysm.

This complication might have been avoided by early recognition of septic loosening of the THA followed by

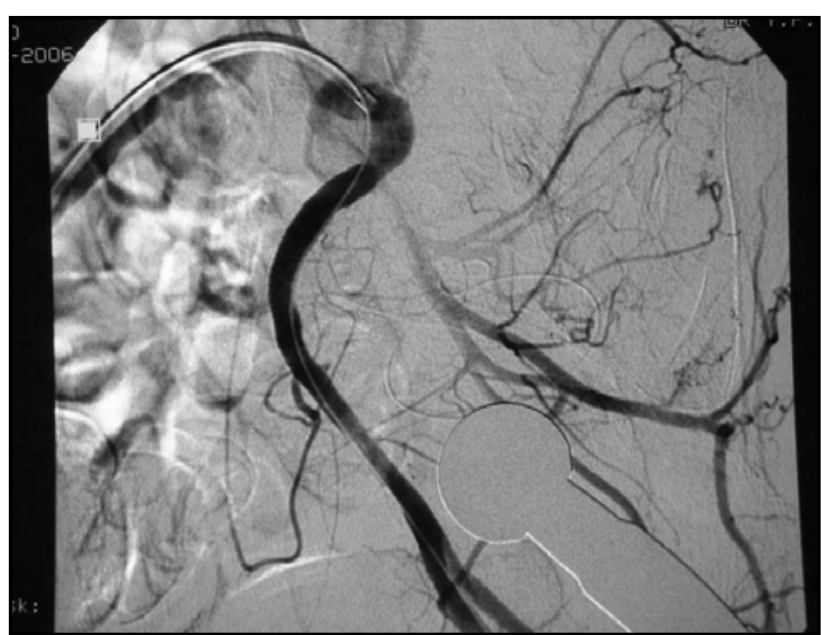

Fig. 2: A digitally enhanced and subtracted image of an angiogram of the external iliac artery showing a large pseudoaneurysm of the external iliac artery in close proximity to the acetabular component and implanted femoral head.

appropriate revision surgery to prevent migration of acetabular component. The case serves as a reminder that in THA revision, it is important to consider the possibility of the existence of an aneurysm or pseudoaneurysm, especially in cases where there is a migrated acetabular component.

Bach et $\mathrm{l}^{3}$ reported an injury to the external iliac artery from an acetabular cup which resulted in formation of a pseudoaneurysm. They believed that the threaded acetabular cup with sharp cutting flutes might have caused direct lesion of the arterial wall. Although the acetabular component used in this patient did not have sharp cutting flutes, we believe that the arterial lesions might have been similarly caused by repetitive direct trauma from either the acetabular cup or the implanted femoral head.

\section{CONCLUSION}

Vascular complications associated with THA are remarkably rare, making diagnosis and treatment of such sequelae extremely challenging for surgeons who are not familiar with their management (as evidenced by the high rate of limb loss, $70 \%$ ). This case demonstrates that a pseudoaneurysm does not always manifest as an acute presentation secondary to direct injury during a surgical procedure. It can present late, and be caused by repetitive trauma from arthroplasty components secondary to septic loosening. A pseudoaneurysm can threaten both the survival of the affected limb and the life of the patient. It is therefore important to recognise the early signs and symptoms of infected arthroplasty in order to prevent such complications. If a pseudoaneurysm is suspected, an angiogram should be performed followed by appropriate treatment as soon as possible. 


\section{REFERENCES}

1. Nachbur B, Meyer RP, Verkalla K, Zürcher R. The mechanism of severe arterial injury in surgery of the hip joint. Clin Ortho 1979; 141: 122-33.

2. Sethuraman V, Hozack WJ, Sharkey PF, Rothman RH. Pseudoaneurysm of femoral artery after revision total hip arthroplasty with a constrained cup. J Arthroplasty 2000; 15(4): 531-4.

3. Bach CM, Steingruber I, Wimmer C, Ogon M, Frischhut B. False aneurysm 14 years after total hip arthroplasty. J Arthroplasty 2000; 15(4): 535-8. 